\title{
UM QUADRO DE REFERENCIA ENTRE OS PRINCIPAIS MODELOS DE ESTRATÉGIA DE OPERAÇÕES E AS SUAS APLICABILIDADES NO SETOR SIDERÚRGICO NACIONAL
}

\section{A REFERENCE FRAMEWORK BETWEEN THE MAIN MODELS OF STRATEGY OF OPERATIONS AND THEIR APPLICABILITY IN NATIONAL STEEL SECTOR}

Data do recebimento do artigo: 13/09/2012

Data do aceite do artigo: 11/3/2013

Data da publicação: 10/6/2013

\author{
Anselmo Alves Bandeira ${ }^{1}$ \\ Doutor em Engenharia de Produção \\ Escola Politécnica da Universidade Federal da Bahia \\ Carlos Alberto Safatle \\ Doutor em Administração \\ Pontifícia Universidade Católica de São Paulo
}

\section{RESUMO}

O artigo aborda a avaliação de desempenho como sendo decorrente da estratégia competitiva. Contendendo os conceitos e os modelos relativos à formulação estratégica de manufatura, procuramos desdobrar um procedimento sistemático capaz de interagir os níveis de desempenho operacionais e não operacionais ao desenvolvimento da estratégia competitiva. No que concerne à abordagem de pesquisa quantitativa, utilizamos a análise de séries temporais à interpretação dos resultados logrados, concebendo o setor siderúrgico brasileiro como a unidade de análise deste estudo de caso real. Procuraremos descrever o cenário internacional, realçando as tendências e caracterizando a unidade de análise dentro deste contexto. $\mathrm{O}$ artigo tem como proposta o detalhamento de uma temática ainda pouco perscrutada na literatura, assim como a exploração dos meios para conduzir uma organização de forma pró-ativa e com efetividade aos seus objetivos corporativos.

Palavras-Chaves: Gestão de Operações, Estratégia de Operações, Competição, Manufatura, Medida de Desempenho, Plano de Contingência, Processo de Tomada de Decisão.

\footnotetext{
${ }^{1}$ Autor para correspondência: Universidade Federal da Bahia - João Carlos Salles Pires da Silva. Endereço: Rua Augusto Viana , s/n - Palácio da Reitoria , Canela , 40110-909, Salvador.
} 


\begin{abstract}
This paper aims to appraise performance as a process that stems from the employment of a competitive strategy. By pitting the concepts and models relative to the manufacturing strategy formulation against each other, it seeks to unfold a systematic process capable of fostering interaction between operational and non-operational levels and the development of competitive strategy. As the approach to interpret the results obtained is based on a statistical analysis, the time-series analysis is used. The study seeks to look into the means to run an organization proactively and effectively, so as to reach its corporate goals.
\end{abstract}

Keywords: Operations Management, Operations Strategy, Competition, Manufacturing, Performance Measurement, Contingency Plan, Decision-Making Process. 


\section{INTRODUÇÃO}

Na década de 1960, a abordagem do planejamento incidia sobre as ferramentas e as técnicas que auxiliavam os "tomadores de decisão" a administrarem o negócio. No decênio de 1970, o foco era sobre os compromissos financeiros, dando margens às estratégias alternativas. No decenário de 1980, explorou-se como as organizações deveriam perceber a necessidade de mudança de estratégia (WHITTINGTON, 1996), (BONN; CHRISTODOULOU, 1996), FROHLICH; WESTBROOK (2001) e ROBERTS (2001). Neste final de década, a tendência dos novos paradigmas converge para a competição baseada no tempo.

STALK (1988) propende uma das principais razões do baixo desempenho em uma competição baseada no tempo como sendo a defasagem entre a variação de demanda e o tempo de resposta, função do sistema de manufatura flexível. Neste prisma, podemos suplementar, predizendo que as estratégias pioneiras apresentam um grau de incerteza maior, todavia, com maior potencial de retorno.

GOLD (1973) aponta as inovações tecnológicas como inerentemente atrativas por convergirem em recompensas econômicas, motivo que sustenta os altos investimentos e os desenvolvimentos de programas que repercutem em lucros brutalmente desiguais em proporção ao investimento realizado. Quanto maior a complexidade do sistema, maior é a disparidade entre o lucro e o investimento, maior o índice de produtividade, menor o custo unitário, maior a lucratividade e o crescimento da organização, frisa o autor.

Inúmeras organizações operam, hoje, em ambientes turbulentos que requerem estratégias complexas, que terminam sendo válidas para uma certa circunstância e que perdem a validade quando se mudam as condições de contorno. Para estes ambientes, o entendimento das relações de causa e efeito torna-se mais do que uma questão de sobrevivência e sim um diferencial competitivo. Prover de uma estratégia competitiva é a única forma de caucionar uma posição unívoca e diferenciada.

A literatura trata as medidas de desempenho essencialmente em duas fases: antes e depois da década de 1980. Na fase anterior, a ênfase consistia em medidas financeiras tal como lucro por unidade de produção, retorno sobre o investimento, retorno sobre vendas, variações de preço, vendas por funcionário e produtividade.

$\mathrm{Na}$ fase posterior, em conseqüência da redução da participação de mercado para os concorrentes, capazes de oferecer produtos de menor custo, de melhor qualidade, em menor tempo e com maior variedade, as organizações procuraram implementar novas tecnologias e filosofias de gestão da produção. Por exemplo: CIM (Computer Integrated Manufacturing), FMS (Flexible Manufacturing System), JIT (Just in Time), OPT (Optimized Production Technology), TQM (Total Quality Management) etc. 
As medidas de desempenho tradicionais normalmente não circunscrevem a estratégia defendida pela organização de forma clara, propendendo em minimizar custos, em elevar o nível de eficiência da força de trabalho, em otimizar a utilização de máquinas, enfim. Existe também uma inflexibilidade em se ajustar estas medidas cruzando todas as áreas de decisão, dificultando a mensuração da efetividade2 da organização (DATTA, 1996), (POST, 1997) e (SLACK, 1993).

\section{OBJETIVO}

Este artigo tem por objetivo principal a discussão de um procedimento sistemático de avaliação de desempenho sintonizado à gestão estratégica, capaz de interpretar o comportamento do sistema de operações e fornecer informações inteligíveis ao processo de formulação da estratégia da organização e à monitorização do desempenho.

O foco do trabalho relata a conclusão sumária de um estudo de caso real do setor siderúrgico brasileiro, desenvolvido em uma tese de doutorado na Escola Politécnica da USP, através da análise de indicadores de desempenho em um intervalo de tempo previamente determinado, possibilitando, assim, a interpretação dos resultados obtidos.

\section{QUADRO COMPARATIVO DOS MODELOS: VANTAGENS E DESVANTAGENS}

Em prol da vasta revisão bibliográfica, torna-se imprescindível relatar apenas as principais vantagens e desvantagens das proposições e dos modelos, convergindo aqueles mais próximos para a atual contingência do setor siderúrgico nacional.

\section{Proposições}

A proposição de GHALAYINI; NOBLE (1996) tem como principal característica a diferenciação entre medidas tradicionais e não tradicionais. As medidas tradicionais terminam restringindo a flexibilidade das organizações quanto ao restabelecimento de sua efetividade, defasam o sistema de informação, segregam-se do sistema de operações, dificultam o acompanhamento da estratégia competitiva, estabelecem fronteiras entre as funções da organização etc.

As medidas de mercado promovem maior aderência na retroalimentação das informações, ao passo que as medidas financeiras, por si só, não conduzem a organização ao alcance dos objetivos estratégicos de longo prazo.

\footnotetext{
${ }^{2}$ Significa a qualidade de ser efetivo; condiz à orientação da empresa a longo prazo.
} 
RANGONE (1996) propõe uma hierarquia do problema da decisão que permite rastrear o foco do problema, dentro de uma visão sistêmica, contudo, necessita de uma visão bem estruturada por parte dos "tomadores de decisão", inclusive das repercussões de cada decisão no nível operacional.

A proposição de FITZGERALD et al. (1991) retrata o sistema geral de avaliação de desempenho sob o prisma de médias ou determinantes e resultados. A grande lacuna das médias ou determinantes está no procedimento de quantificar também atributos qualitativos, podendo incorrer em falhas de concepção, mais agravante ainda quando inexiste o histórico.

Ainda mais: os resultados geram consistência na reavaliação do desempenho, uma vez que está calcada em fatos reais, como também pela sua proximidade ao mercado e pela constatação da estrutura da organização.

BITITCI; CARRIE; MCDEVITT (1997) apresentam uma proposição interativa, entre as funções da organização, e iterativa, pleiteando pela abrangência, coerência e consistência. O maior desafio consiste no estabelecimento de parâmetros dos fatores críticos de sucesso para consolidar os planos de ação.

A proposição de KIM; ARNOLD (1996) estabelece as prioridades à manufatura decorrentes da definição da estratégia do negócio e enfatiza a ligação entre a estratégia competitiva e o sistema de produção. A sua essência está no equilíbrio entre as relações das prioridades à manufatura e às decisões estruturais e infra-estruturais.

A grande contribuição da proposição de SKINNER (1978) fundamenta-se no contexto amplo em que são incorporados a estratégia organizacional, as políticas da manufatura, os fatores de produção, a disponibilidade de recursos e o procedimento de reavaliação dos resultados obtidos.

Um ponto muito importante é a consideração das tendências de mercado e do nível competitivo da concorrência. Em contrapartida, podem surgir conflitos na fixação da política de manufatura versus algumas restrições pertinentes aos outros aspectos.

Na verdade, a proposição de GOODMAN; LAWLESS (1994) é substancialmente iterativa, sempre questionando a efetividade da estratégia competitiva implementada, porém não detalhando a ponto de rastrear o foco do problema. Assim, sem uma análise mais acurada, as decisões cabíveis poderão estar desagregadas dos objetivos estratégicos.

As multifaces da estratégia de manufatura propostas por LEONG; WARD (1995) não têm a intensão de ser exaustivas, apresentando vazios como qualidade, sistema de informação, exigências de mercado etc. 
Por fim, a proposição de FINE; HAX (1985) sugere o planejamento da estratégia corporativa segundo uma hierarquia, que parte do nível corporativo, passando pelo nível do negócio até chegar ao nível funcional. 


\begin{tabular}{|c|c|c|c|c|c|c|}
\hline \multicolumn{7}{|c|}{ MODELOS DE ESTRATÉGIA DE MANUFATURA } \\
\hline GARVIN & HILL & CORRÊA E GIANESI & SLACK & PLATTS E GREGORY & MUSCAT & KAPLAN E NORTON \\
\hline $\begin{array}{l}\text { Incorpora as tendências de } \\
\text { mercado. }\end{array}$ & $\begin{array}{l}\text { Mais detalhista na } \\
\text { formulação da estratégia de } \\
\text { manufatura. }\end{array}$ & $\begin{array}{l}\text { Converge com o modelo } \\
\text { de HILL, ao direcionar o } \\
\text { espectro de estratégias à } \\
\text { contingência das funções } \\
\text { de marketing e de } \\
\text { manufatura. }\end{array}$ & $\begin{array}{l}\text { Fornece subsídios à } \\
\text { avaliação da utilização dos } \\
\text { recursos produtivos. }\end{array}$ & $\begin{array}{l}\text { Interpreta as inclinações de } \\
\text { mercado, assim como as } \\
\text { oportunidades e ameaças. }\end{array}$ & $\begin{array}{l}\text { Avalia a importância de } \\
\text { indicadores de } \\
\text { desempenho. }\end{array}$ & $\begin{array}{l}\text { Atenua as divergências } \\
\text { entre as perspectivas: } \\
\text { financeira, do cliente, de } \\
\text { negócio e de } \\
\text { inovação/aprendizado. }\end{array}$ \\
\hline $\begin{array}{l}\text { Aproxima as áreas de } \\
\text { decisão dos critérios } \\
\text { competitivos. }\end{array}$ & $\begin{array}{l}\text { Interage a estratégia de } \\
\text { manufatura à estratégia de } \\
\text { marketing, através de } \\
\text { critérios competitivos. }\end{array}$ & $\begin{array}{l}\text { Contempla os nichos } \\
\text { mercadológicos e a } \\
\text { disponibilidade de } \\
\text { tecnologia da organização. }\end{array}$ & $\begin{array}{l}\text { Permite confrontar a visão } \\
\text { dos “tomadores de } \\
\text { decisão" e a visão do } \\
\text { cliente, canalizando o } \\
\text { esforço de gestão. }\end{array}$ & $\begin{array}{l}\text { Promove potencial de } \\
\text { melhoria, analisando o } \\
\text { nível de desempenho } \\
\text { dentro da concepção do } \\
\text { cliente. }\end{array}$ & $\begin{array}{l}\text { Alia as métricas a partir do } \\
\text { planejamento estratégico, } \\
\text { objetivos corporativos, } \\
\text { cenário competitivo, estilo } \\
\text { empreendedor e do } \\
\text { desenvolvimento de } \\
\text { competências. }\end{array}$ & $\begin{array}{l}\text { Adequado à discussão dos } \\
\text { objetivos corporativos. }\end{array}$ \\
\hline $\begin{array}{l}\text { Orienta os programas e os } \\
\text { projetos. }\end{array}$ & $\begin{array}{l}\text { Demonstra clareza em cada } \\
\text { estágio, dando uma visão } \\
\text { consistente. }\end{array}$ & \multirow[t]{4}{*}{$\begin{array}{l}\text { Não associa com clareza os } \\
\text { modelos de contingência } \\
\text { aos objetivos corporativos. }\end{array}$} & $\begin{array}{l}\text { Não traduz, diretamente, as } \\
\text { relações entre a } \\
\text { importância e o } \\
\text { desempenho de cada } \\
\text { critério com os objetivos } \\
\text { de marketing e de } \\
\text { manufatura. }\end{array}$ & $\begin{array}{l}\text { Determina prioridades às } \\
\text { famílias de produtos } \\
\text { através das exigências de } \\
\text { mercado. }\end{array}$ & $\begin{array}{l}\text { Estabelece relações de } \\
\text { dependência entre as } \\
\text { variáveis do sistema de } \\
\text { produção, facilitando a } \\
\text { monitorização e a predição } \\
\text { de resultados. }\end{array}$ & $\begin{array}{l}\text { Não declara um } \\
\text { procedimento sistemático } \\
\text { para o planejamento do } \\
\text { negócio, nem à formulação } \\
\text { da estratégia competitiva. }\end{array}$ \\
\hline $\begin{array}{l}\text { Auxilia na retificação de } \\
\text { políticas. }\end{array}$ & $\begin{array}{l}\text { Facilita o investimento de } \\
\text { capital para atender os } \\
\text { critérios competitivos. }\end{array}$ & & $\begin{array}{l}\text { Implica na avaliação, a } \\
\text { curto prazo, de soluções de } \\
\text { compromisso. }\end{array}$ & $\begin{array}{l}\text { Sugere a formalidade de } \\
\text { políticas a cada área de } \\
\text { decisão da manufatura. }\end{array}$ & $\begin{array}{l}\text { Não detalha os critérios } \\
\text { competitivos e nem os } \\
\text { associa aos fatores críticos } \\
\text { de sucesso. }\end{array}$ & $\begin{array}{l}\text { Gera um consenso entre a } \\
\text { visão e a estratégia. }\end{array}$ \\
\hline $\begin{array}{l}\text { Não trata a conciliação dos } \\
\text { interesses das áreas de } \\
\text { decisão. }\end{array}$ & $\begin{array}{l}\text { Realça o potencial de } \\
\text { melhoria. }\end{array}$ & & \multirow{2}{*}{$\begin{array}{l}\text { Delimita os planos de } \\
\text { ação, quanto à sua } \\
\text { viabilidade, bem com a sua } \\
\text { vulnerabilidade e sua } \\
\text { receptividade. }\end{array}$} & \multirow{2}{*}{$\begin{array}{l}\text { Adequado à revisão da } \\
\text { estratégia competitiva, } \\
\text { entretanto, falha por não se } \\
\text { associar, diretamente, aos } \\
\text { objetivos de marketing. }\end{array}$} & \multirow{2}{*}{$\begin{array}{l}\text { Não confronta a avaliação } \\
\text { de desempenho com as } \\
\text { características do sistema } \\
\text { de produção. }\end{array}$} & \multirow{2}{*}{$\begin{array}{l}\text { Integra o planejamento } \\
\text { estratégico aos } \\
\text { procedimentos } \\
\text { orçamentários. }\end{array}$} \\
\hline $\begin{array}{l}\text { Indicado para situações em } \\
\text { que haja clareza das } \\
\text { funções da manufatura } \\
\text { diante dos objetivos } \\
\text { estratégicos. }\end{array}$ & $\begin{array}{l}\text { Não evidencia possíveis } \\
\text { variações na concepção } \\
\text { das relações de utilização } \\
\text { de capacidade e estoques } \\
\text { sobre a margem bruta de } \\
\text { contribuição. }\end{array}$ & & & & & \\
\hline
\end{tabular}

Quadro 1 - comparativo dos Modelos de Estratégia de Manufatura: Vantagens e Desvantagens

Fonte: Dados da pesquisa. Elaborado pelos autores. 


\section{CONSIDERAÇÕES FINAIS}

Diante das diversas proposições revisadas na seção anterior, algumas são mais propícias ao setor siderúrgico nacional. A proposição de KIM; ARNOLD (1996), por ser dinâmica quanto ao aspecto das oscilações de mercado, interage o sistema de operações com a estratégia competitiva através da estratégia do negócio e das prioridades à produção, influenciando as decisões sobre o planejamento, a utilização de capacidade, a tecnologia, os níveis de desempenho operacional etc.

Outra proposição cabível ao setor é a de FINE; HAX (1985) por estabelecer uma hierarquia do planejamento, considerando os nichos mercadológicos e as famílias de produtos; por exigir um posicionamento estratégico e por vincular os procedimentos orçamentários à formulação da estratégia competitiva. Esta proposição demonstra ser a mais próxima da atual contingência do setor.

Atinente aos modelos, isoladamente, o modelo de HILL (1995) presume ser o mais completo à formulação da estratégia de manufatura. Porém, a junção de alguns modelos certamente dará mais estrutura ao desenvolvimento e à sustentação da estratégia competitiva, principalmente em um setor que depende de seus resultados em uma economia globalizada.

Pode-se até utilizar, como suporte, o modelo de SLACK (1994) para esboçar o perfil do cliente ou, se precisar ir além, fazer prospecções de mercado, assim como utilizar o modelo de KAPLAN; NORTON (1992) para discutir os objetivos corporativos a fim de fornecer maiores informações ao modelo de HILL (1995).

De fato, a tônica gira em torno da interligação entre o modelo de HILL (1995), através dos critérios competitivos, com o modelo de PLATTS; GREGORY (1990), por meio das melhorias potenciais identificadas pelas auditorias.

Como toda estratégia competitiva tem que ser implementada e validada, o modelo de MUSCAT (1993) pode ser agregado aos modelos de HILL (1995) e de PLATTS; GREGORY (1990) no intuito de estruturar o desenvolvimento de métricas não tradicionais a fim de monitorizar o sistema de produção e interagir no processo de formulação da estratégia competitiva.

Cabe ressaltar que o procedimento estatístico, como forma de análise do histórico das operações, em confronto com as características do sistema de produção, dará maior consistência à interação do sistema de produção com o processo de formulação da estratégia competitiva.

A literatura contempla a avaliação de desempenho e a gestão estratégica de uma maneira causal3, no entanto, não ostenta um procedimento analítico e sistemático capaz de integrá-los de modo altamente interativo, como é a proposta deste artigo.

Por conseguinte, é plausível sustentarmos um procedimento cíclico cujo objetivo é intercalar o desempenho do sistema de produção na formulação da estratégia de

${ }^{3}$ Para maiores informações, consultar BANDEIRA (1997).

ENIAC Pesquisa, Guarulhos (SP), p. 52-63, v. 2, n. 1, jan.-jun. 2013. 
manufatura, o que é complementário, em termos amplos, aos modelos insculpidos neste artigo. Então, nesta tênue, temos:

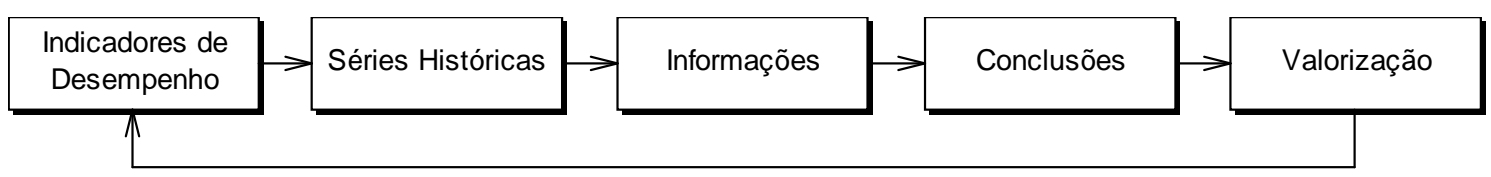

Figura 1 - Retroalimentação da avaliação de desempenho

Fonte: Dados da pesquisa. Elaborado pelos autores.

A integração entre a avaliação de desempenho e a gestão estratégica deve ser aportada segundo os enfoques estratégico e operacional. As informações são provenientes da análise estatística perante o conteúdo da gestão estratégica e o contexto em que a organização se depara.

A estrutura deste trabalho permite desenvolver um sistema de avaliação de desempenho apto a sustentar os critérios competitivos das organizações, reformulando toda a sua concepção com base nas indicações operacionais e não operacionais.

Sua fundamentação é firmada pela consideração do histórico das operações e pela sua previsibilidade, características latentes que propiciam a antecipação de resultados futuros ou até mesmo o rearranjo de possíveis eventos, essenciais ao processo decisório.

Suplementando, conhecendo com profundidade as leis de dependência ou causalidade entre os indicadores de desempenho, é possível acompanhar aqueles que exercem maior influência sobre os demais (principais indicadores ou direcionais de negócio), projetando um cenário mais realista à formulação da gestão estratégica. Sob esta óptica, torna-se possível até o procedimento de simulações, ancorado no conhecimento destas relações causais.

As conclusões são afetadas pelas premissas estabelecidas durante a estruturação dos indicadores de desempenho, como, por exemplo, a distinção entre custo fixo e custo variável, critérios de rateio, nível de rastreamento de cada indicador, enfim.

A força de trabalho, a princípio, é tratada como custo variável, todavia, em um prazo relativamente curto, pode ser considerada como custo fixo. O que define a classificação dos custos também condiz com a tomada de decisão.

A valorização corresponde à definição de uma medida de utilidade em sinergia com a estratégia competitiva e os fatores críticos de sucesso, podendo ou não ser expressa em unidade monetária. Esta valorização é a grande responsável pela avaliação da efetividade organizacional e pela consistência da tomada de decisão, haja vista a conglutinação com as exigências de mercado. 
Assim, o processo de tomada de decisão deverá estar mais sólido, uma vez que a redefinição dos objetivos de desempenho da organização também estará calcada na valorização. Esta redefinição poderá interferir sobre os critérios competitivos da manufatura, causando maior ou menor repercussão, a depender da natureza do plano de contingência.

No que concerne à unidade de análise, o comportamento dos indicadores está, a priori, relacionado ao comportamento da organização (características peculiares), todavia, há indícios de que os resultados logrados podem ser analiticamente generalizáveis, extrapolando as fronteiras da unidade de análise e interpretando, sob o mesmo enfoque, em outros segmentos da economia.

Quanto à generalização estatística, podemos até predizer que é inviável, mesmo porque não se trata do objetivo deste trabalho. É pertinente salientar que, tratando-se de um estudo de caso, há uma tendência para abarcar generalizações a um nível estatístico, o que poderia induzir a problemas de validade externa. A revisão da literatura e o procedimento de análise - análise séries temporais - potencializam os acréscimos da confiabilidade e da validade deste artigo.

Diante deste contexto, esta análise focaliza as relações entre as variáveis de produção do setor siderúrgico nacional, interagindo as informações obtidas com a formulação da gestão estratégica. Outras variáveis também foram analisadas - com por exemplo, fatores econômicos, variação de demanda, tendências de mercado, lucratividade, gestão da cadeia de fornecedores, níveis de estoque, entre outras, identificando o nível de impacto sobre os fatores críticos de sucesso e os critérios competitivos. A abordagem deste artigo incide sobre a função produção, considerando ser a grande força para conduzir uma organização aos seus objetivos corporativos.

\section{REFERÊNCIAS}

BANDEIRA, A.A. Rede de indicadores de desempenho para gestão de uma usina hidrelétrica. São Paulo, 1997. 157p. Dissertação (Mestrado)-Departamento de Engenharia de Produção, Escola Politécnica da Universidade de São Paulo.

BITITCI, U. S.; CARRIE, A. S.; MCDEVITT, L. Integrated performance measurement system: a development guide. International Journal of Operations \& Production Management, Bradford, v.17, n.5, p.522-34, 1997.

FINE, C. H.; HAX, A. C. Manufacturing strategy: a methodology and an illustration. Interfaces, Providence, v.15, n.6, p.28-46, Nov./Dec. 1985.

FITZGERALD, L. et al. Performance measurement in service business. London, cima, 1991. 
FROHLICH, M. T.; WESTBROOK, R. Arcs of integration: an international study of supply chain strategies. Journal of Operations Management, Oxford, v.19, n.2, p.185200, 2001.

GARVIN, D. A. Competing on the eight dimensions of quality. Harvard Business Review, Boston, v.65, n.6, p.101-9, Nov./Dec. 1987.

GHALAYINI, A. M.; NOBLE, J. S. The changing basis of performance measurement. International Journal of Operations \& Production Management, Bradford, v.16, n.8, p.63-80, 1996.

GOODMAN, R. A.; LAWLESS, M. W. Technology and strategy: conceptual models and diagnostics. New York, Oxford University, 1994.

HILL, T. Manufacturing strategy: text and cases. London, Macmillan, 1995.

KAPLAN, R. S.; NORTON, D. P. The balanced scorecard: measures that drive performance. Harvard Business Review, Boston, v.70, n.1, p.71-9, Jan./Feb. 1992.

KIM, J. S.; ARNOLD, P. Operationalizing manufacturing strategy: an exploratory study of constructs and linkage. International Journal of Operations \& Production Management, Bradford, v.16, n.12, p.45-73, 1996.

LEONG, G. K.; WARD, P. T. The six ps of manufacturing strategy. International Journal of Operations \& Production Management, Bradford, v.15, n.12, p.32-45, 1995.

MUSCAT, A. R. N. Modelo para integração de decisões sobre sistemas de operações. São Paulo, 1993. 258p. Tese (Doutorado) - Departamento de Engenharia de Produção, Escola Politécnica da Universidade de São Paulo.

PLATTS, K. W.; GREGORY, M. J. Manufacturing audit in the process of strategy formulation. International Journal of Operations \& Production Management, Bradford, v.10, n.9, p.5-26, 1990.

PROCHNO, P. J. L. C.; CORRÊA, H. L. The development of manufacturing strategy in a turbulent environment. International Journal of Operations \& Production Management, Bradford, v.15, n.11, p.20-36, 1995. 
RANGONE, A. An analytical hierarchy process framework for comparing the overall performance of manufacturing departments. International Journal of Operations \& Production Management, Bradford, v.16, n.8, p.104-19, 1996.

ROBERTS, E. B. Benchmarking global strategic management of technology. Research Technology Management, Bradford, v.44, n.2, p.25-36, 2001.

SKINNER, W. Manufacturing in corporate strategy. New York, John Wiley, 1978.

SLACK, N. The importance-performance matrix as a determinant of improvement priority. International Journal of Operations \& Production Management, Bradford, v.14, n.5, p.59-75, 1994. 